\title{
Write an Original Paper
}

\author{
DJ Lockey, PJF Baskett
}

\section{Introduction}

This article is aimed at trainees who are contemplating writing a short original paper. We aim to cover some of the common pitfalls which can impede smooth progress from good idea to published end product. Although many of the principles discussed here hold for case reports and major papers, the former will be covered in another article in this journal and the latter are best only undertaken with appropriate supervision.

\section{Why bother?}

Cynics often suggest that far too many short papers are published which add little to medical knowledge. There may be some truth in this, but there are several arguments against this standpoint. Firstly, a short paper may be all that is required to present a valid point convincingly. It is not always necessary to prove a point conclusively - a short paper may stimulate a definitive larger study. Also short papers often lead to minor but significant changes in clinical practice. From an author's point of view there are many potential benefits. Preparation and writing is an academic exercise that develops an awareness of the difficulties of trial design and the demands of ethical approval. Practical application of literature search techniques, reference management and the ability to write in a clear, concise and stylish manner are essential skills, not only in paper production, but also in everyday clinical practice. The obvious benefits of curriculum vitae enhancement are particularly important where competition for posts is high. The work done on a paper can also sometimes be used to enhance other areas of a CV, for example, by making presentations and performing audit in the same subject area.

Maj DJ Lockey

FRCA FIMC RCS

(Ed) RAMC (V)

Department of

Anaesthesia

Frenchay Hospital

Bristol BS16 1LE

Email:

djlockey@hotmail.com

Dr PJF Baskett

FRCA FRCP FFAEM

Stanton Court,

Stanton St Quintin,

Nr Chippenham, Wilts

SN14 6DQ

\section{Choice of subject matter}

Straightforward and simple subject matter is highly desirable. Ideally the study should aim to answer a simple question. It is, for example, better to ask if a particular intervention has a particular effect than to look at all of the possible effects of that intervention. Often the initial idea comes from someone who will make a suitable supervisor. It should certainly be discussed with someone with a successful track record of publication. A subject in which the investigator is particularly interested is preferable. There is nothing worse than being allocated a study in which the investigator will invest considerable effort but has no interest from the outset. It is much less likely to be finished. Some things are much easier to investigate than others. If large numbers of patients are required to produce a study it is no good looking at a condition which is only seen rarely in the proposed clinical environment - it may take years to complete the study. Time is a huge consideration for trainees. In trainee clinical posts it is rare to spend long periods in the same location. This means that completion of a study is often very difficult in the time available. It is vital to be realistic about how long things will take. It is usually undesirable to pass on half finished projects to successors. Conversely, taking on a good project which has been well thought out and already received ethical approval might be practical. It is essential to read papers in the journals in which one is aiming to publish. It will rapidly become apparent that papers of similar impact and length have had hugely variable amounts of work put into them. It is important only to take on a project if you are confident that you can complete it. Half-finished, poorly conducted projects are a waste of everyone's time and, if patients are involved, are also unethical.

\section{Supervisors}

An ideal supervisor is one who has a record of successful publication, knows how much time is available (and how much can be achieved realistically within it), recognises your writing level (or lack of it), and is available and approachable. They are a rare breed. It should be remembered that successful publication is usually also of benefit to the supervisor since it shows evidence of continuing professional development and enhances (now compulsory) personal portfolios.

\section{Study design}

Time taken to understand a little about the design of studies is time well spent. Of particular importance are randomisation and blinding techniques, control groups and power studies. It pays to make the study as high quality as possible at the outset. Finding out after study completion that the study groups were different in more than the variable being studied can severely weaken its impact. A medical statistician should be consulted at this stage and the study design discussed. Medical statisticians often sit on ethical committees and the project is less likely to be approved if there are basic 
statistical flaws in the study design that could have been avoided by early consultation.

\section{Patients and ethical approval}

If patients are involved in the study there will inevitably be delays. Ethical approval can take months. It is important to find out when the next ethics committee meeting is and have the necessary forms ready for consideration, the next meeting may be months away. In order to avoid rejection, time should be taken to fill in the ethics forms carefully. It should be remembered that lay people sit on the committee and so technical issues should be made as clear as possible. This is particularly important for patient information sheets which should be very clear. An example might be "a plastic tube is then placed into your windpipe (trachea)" rather than "the trachea is then intubated". The protocol can be "trialled" on a non-medical relative of appropriate age. If they don't understand, do it again. Sometimes if a study is uncontroversial, (which often means that no additional interventions are carried out on patients recruited into the study), 'Chairman's approval' can be requested. This means that the Chairman of the ethical committee has read and accepted your proposal and that you may begin but are still subject to the decisions of the full committee after it meets.

\section{Data Collection}

If colleagues are being expected to collect raw data, it is essential to make sure that the data forms are easy to fill in and as short as possible. Forms must be checked and the data collectors talked to in order to ensure that a consistent approach is being taken. Some journals expect raw data to be kept for a number of years so that they can be examined in the event of inconsistencies. The compilers of Meta analyses may also require original data at some time in the future. The data should be entered on a computer database (Excel or Access are suitable depending on amount and complexity). It is important to remember the rules about putting patients on databases and always make data anonymous.

\section{Writing the paper}

There are basic rules when writing a paper which vary slightly depending on the journal to which you are submitting. It is vital to get the journal that you hope to publish in and to find and master the 'instructions for authors' section. This is particularly useful in determining the length of the paper. Papers are more often returned for being too long than too short, so if the message can be got across in fewer words, it should be. Most people want their paper published in the best journal that will accept it. Journals have impact factors calculated on the basis of the number of citations that papers in that particular journal get. It is helpful to think of the intended readership. If only people in one's own specialty are likely to be interested in what one has to say, time should not be wasted sending the article to a very general journal. Sending a poorly prepared paper to an inappropriate journal will result in it being rejected and this will waste a considerable amount of time (several months at least).

The paper is usually divided into sections, each of which has a specific purpose. They are:

- title and abstract

- introduction

- methods

- results

- discussion

\section{Title and Abstract}

The title and abstract give the reader a chance to assess whether the paper is worth reading. The title should indicate something about the design of the study for example "A randomised controlled trial of..." or "An observational study of...". The abstract gives the key points in every section and is limited strictly to $200-250$ words. It is the abstract that is used to summarise the published paper in literature search systems such as "Medline". Structured abstracts are often requested and the guidelines must be followed carefully.

\section{Introduction}

The introduction section should explain why the study was carried out. It may summarize current knowledge in the subject area briefly and show why the study needed to be performed to fill a knowledge gap. It may contain references. A clear question or statement of intent at the end of the introduction is helpful to the reader.

\section{Methods}

This section explains how the study was carried out. It should be as short as possible but contain all essential information. This includes a description of who the recruited subjects were, how they were recruited and demographic details such as age and sex distribution. This information is essential because it allows the reader to judge whether the results are applicable to a wider population. Techniques applied in the protocol, methods of data collection and statistical systems used are described. The methods section plays an important part in the assessment of study quality.

\section{Results}

The results of the study should be presented in a way which allow readers to interpret the findings for themselves. This often means the presentation of numbers in easy to understand tables in a logical manner. The text in the results section gives the author's approach to the results but without discussion of their relevance (which should be reserved for the discussion section). 


\section{Discussion}

This section answers the question 'What do the results mean?' It should also comment on the applicability of the study to the wider population. Discussion of the limitations of the study may be included. Most studies have limitations and it is often better to be open about them and to show that they have been considered rather than to have to defend them in correspondence after publication. Other relevant studies should be quoted in this section and the implications of the study made clear in relation to them. Ideally the study should have answered the question set in the introduction and hopefully confirmed or suggested changes in clinical practice. This is often not the case but if useful information is presented the study may still be published. As mentioned above, a common mistake, which is invariably picked up at review stage, is to include results in the discussion and vice versa. The two sections must be rigourously separated.

\section{References}

References can be managed manually or with commercially available reference manager systems. Reference managers are particularly helpful where there are large numbers of references (often the case with review articles) or when the author is writing multiple papers in the same subject area (and therefore often uses the same references).

If managing references by hand, an efficient method is to leave the full references in the text (bracketed) and only extract them when the paper is completed. This means that if a draft is altered and a reference changed or added the whole numbering system does not have to be altered each time. The format of references is important and will be stated in 'instructions for authors' in the specific journal. The most common format is Vancouver and references can usually be downloaded directly from literature searches in this form.

\section{The Editorial Process}

The first thing that the editor will do is to check that the paper is submitted in the stated style of the journal with the appropriate number of hard copies and a disc or other electronic format. All authors are required to sign the submission letter. If patients are involved, ethical approval must have been obtained.

The editor will scan the paper briefly to ensure that the content is potentially publishable and relevant to the journal. $\mathrm{He}$ or she will then send a copy to at least two reviewers with a known interest in the subject with a deadline of, normally, 4-6 weeks to reply. Good reviewers are often very busy people and here a delay can occur. Most journals ask reviewers to return the paper immediately if they cannot complete the task on time but, on occasion, delays occur through failed, but good, intentions. The reviewers' reports are usually presented in two sections - one for the authors and one for the editor's eyes only. The latter, but not the former, will contain a recommendation as to whether the paper should be published as it is, with minor changes, be reconsidered after major changes, or rejected. The editor's job is to assess the reviewers reports and decide on the category of response to the authors. In most journals the reviewers' names are not revealed to the authors. After the reviewers reports have been returned to the authors they should make the necessary changes to comply with the reviewers comments, or make a very good case as to why this should not, or cannot, be done.

The editor will now review the revised paper in the light of the reviewers comments and, perhaps, his own opinion. He will go through the paper line by line for accuracy, good English and presentation and then send it off for publication, perhaps with a note on the priority required. The authors will be informed that this has occurred. At the publishing house the professional copy editor will check the English once again and search for typographical errors and reference accuracy. Colour illustrations, if the journal offers this facility, may cost more and may be charged to the authors.

The editor and the authors receive a proof for checking when publication is imminent. Proofs must be returned in a matter of days otherwise publication may be delayed.

The editorial process usually takes 6-9 months from submission to appearance in print. Many factors affect this time including the popularity of the journal amongst potential authors, the reviewers' adherence to deadlines, and timely response by the authors to their comments. Most journals have a website which can give an indication of the progress of the paper after it has reached the publisher.

\section{After Publication}

After the paper has been published, it is important to maximise the effort which you have put in. Findings may be presented at departmental, local, regional or national meetings. This may generate the publication of abstracts in the proceedings of the meetings. It is interesting to watch out for correspondence in subsequent issues of the journal and to reply in a clear and considered manner.

\section{Conclusion}

Writing short papers can be a valuable exercise. To have the maximum chance of publication in a good journal, considerable investment should be made in the planning stages. Careful attention to 'instructions for authors' and a systematic approach to writing (as described) make acceptance much more likely. 